\title{
Further Hopping with Toads and Frogs
}

\author{
Thotsaporn "Aek" Thanatipanonda \\ Research Institute for Symbolic Computation (RISC) \\ Johannes Kepler University, A-4040 Linz, Austria \\ thotsaporn@gmail.com
}

Submitted: Jun 15, 2009; Accepted: Mar 17, 2011; Published: Mar 24, 2011

Mathematics Subject Classification: 91A46

\begin{abstract}
We prove some new results about the combinatorial game "Toads and Frogs". We give a finite number of recurrence relations for computing the values of all positions with exactly one $\square$. We show that $\mathrm{T}^{a} \square \square \mathrm{F}^{a}$ is an infinitesimal for $a \geq 4$. At the end, we make five new conjectures and describe possible future work.
\end{abstract}

\section{Introduction}

The game Toads and Frogs, invented by Richard Guy, is extensively discussed in "Winning Ways" [1], the famous classic by Elwyn Berlekemp, John Conway, and Richard Guy, that is the bible of combinatorial game theory.

The game is played on a $1 \times n$ strip with either $\operatorname{Toad}(\mathrm{T})$, $\operatorname{Frog}(\mathrm{F})$ or $\square$ on the squares. Left plays $\mathrm{T}$ and Right plays $\mathrm{F}$. $\mathrm{T}$ may move to the immediate square on its right, if it happens to be empty, and F moves to the next empty square on the left, if it is empty. If $\mathrm{T}$ and $\mathrm{F}$ are next to each other, they have an option to jump over one another, in their designated directions, provided they land on an empty square [1, p.14]. Throughout the paper, we will use the notation $X^{n}$ to denote $n$ contiguous copies of the Toads and Frogs position $X$. For example, $\square^{3}(\mathrm{TF})^{2} \mathrm{~F}$ is shorthand for $\square \square \square$ TFTFF.

Already in [1] there is some analysis of Toads and Frogs positions, such as TT $\square$ FF $\square$ and $\square^{a} \mathrm{TF} \square^{b}$. In 1996, Erickson [2] analyzed more general positions, and made six conjectures about the values of some families of positions. All of them are starting positions (positions where all $\mathrm{T}$ are rightmost and all $\mathrm{F}$ are leftmost). Erickson's conjectures were:

E1: $\mathrm{T}^{a} \square \square \mathrm{F}^{b}=\{\{a-3 \mid a-b\} \mid\{* \mid 3-b\}\}$ for all $a>b \geq 2$.

$\mathrm{E} 2: \mathrm{T}^{a} \square \square \square \mathrm{FF}=\{a-2 \mid a-2\}$ for all $a \geq 2$.

E3: $\mathrm{T}^{a} \square \square \square \mathrm{FFF}=a-\frac{7}{2}$ for all $a \geq 5$.

E4: $\mathrm{T}^{a} \square^{a} \mathrm{~F}^{a-1}=1$ or $\{1 \mid 1\}$ for all $a \geq 1$. 
E5: $\mathrm{T}^{a} \square^{b} \mathrm{~F}^{a}$ is an infinitesimal for all $a, b$ except $(a, b)=(3,2)$.

E6: Toads and Frogs is NP-hard.

Jesse Hull proved E6 in 2000 [3]. Doron Zeilberger and the author proved E2 in [4]. The proofs of Conjectures E1 and E3 are in [5]. The results of this paper include a counterexample to E4. Conjecture E5 is still open.

This paper is a sequel to [4], which discusses the symbolic finite-state approach to prove the value of the positions in class Aij and Bij (defined below). However, the nice formulas of the value of Toads and Frogs game are not limited to only class Aij or class $\mathrm{B} i j$. There are also nice formulas in the positions where variables are on both $\mathrm{T}$ and $\mathrm{F}$, for example $\mathrm{T}^{a} \square \square \mathrm{F}^{b}$. In this paper we analyze some of these positions.

\section{Definitions:}

Class Aij: All positions that have exactly $i$ occurrences of $\square$ and exactly $j$ occurrences of F. In other words, the positions where for each of the permutation of $i$ of $\square$ and $j$ of $\mathrm{F}$, we insert a variable (symbolic) number of $\mathrm{T}$ in-between the $\square$ and F. For example, A11 is all positions with one $\square$ and one $\mathrm{F}$. These positions are $\mathrm{T}^{a} \square \mathrm{T}^{b} \mathrm{FT}^{c}$ and $\mathrm{T}^{a} \mathrm{FT}^{b} \square \mathrm{T}^{c}$, $a \geq 0, b \geq 0$ and $c \geq 0$.

Class Bij: All positions that have exactly $i$ occurrences of $\mathrm{T}$ and exactly $j$ occurrences of F. In other words, the positions where for each of the permutation of $i$ of $\mathrm{T}$ and $j$ of $\mathrm{F}$, we insert a variable (symbolic) number of $\square$ in-between the $T$ and F. For example, B11 is all positions with one $\mathrm{T}$ and one $\mathrm{F}$. These positions are $\square^{a} \mathrm{~T} \square^{b} \mathrm{~F} \square^{c}$ and $\square^{a} \mathrm{~F} \square^{b} \mathrm{~T} \square^{c}$, $a \geq 0, b \geq 0$ and $c \geq 0$.

General Class A $i$ : all positions that have exactly $i$ occurrences of $\square$. In other words, the positions of the general class $\mathrm{A} i$ are $X_{1} \square X_{2} \square \ldots X_{i} \square X_{i+1}$, where $X_{k}:=\mathrm{T}^{a_{1}} \mathrm{~F}^{a_{2}} \mathrm{~T}^{a_{3}} \mathrm{~F}^{a_{4}} \ldots$, $a_{1}, a_{2}, a_{3}, \cdots \geq 0$.

General Class B $i$ : all positions that have exactly $i$ occurrences of $\mathrm{F}$. In other words, the positions of the general class $\mathrm{B} i$ are $Y_{1} \mathrm{~F} Y_{2} \mathrm{~F} \ldots Y_{i} \mathrm{FY} Y_{i+1}$, where $Y_{k}:=\mathrm{T}^{a_{1}} \square^{a_{2}} \mathrm{~T}^{a_{3}} \square^{a_{4}} \ldots$, $a_{1}, a_{2}, a_{3}, \cdots \geq 0$.

Note: we need exactly $\left(\begin{array}{c}i+j \\ j\end{array}\right)$ functions to represent all positions in the class A $i j$ and $\mathrm{B} i j$.

Class Aij and Bij are discussed in [4]. We develop the new approach called "the finite state method" to prove all values of positions that occur in each of these classes. The general class $\mathrm{A} i$ and the general class $\mathrm{B} i$ are the generalization of class $\mathrm{A} i j$ and $\mathrm{B} i j$ since now we have no constraint on the fixed number of either $\square$ or F as in Aij and Bij. Unlike the class Aij, these classes are much harder to categorize the values of all positions. Here we can not apply the finite state method used in [4] anymore, since we have infinitely many positions that come from the combination of other two letters with symbols on them ie. $X_{k}$ and $Y_{k}$. The general class A1, the class of all positions with exactly one $\square$, is the only general class that we are able to categorize all positions.

Many positions in these general classes do not have a nice compact formula, formula that has a fixed shape; for example in $\mathrm{A} 2, \mathrm{~T}^{a} \square \mathrm{TF} \square \mathrm{TF}^{b}$. On the other hand, many positions have a nice compact formula. Once we detect the patterns of the positions, the 
proof is quite routine. We then do the proof of each specific position with the help of a computer program. More results of these types of problem are in [5]. We hope to see the computer playing more roles in assisting with proofs in the future.

The main results in this paper are

Theorem 1. There is a finite number of recurrence relations for the values of positions in general class A1, any position with one $\square$.

Theorem 2. $\mathrm{T}^{a} \square \square \mathrm{F}^{a}$ is an infinitesimal, $a \geq 4$.

Theorem 1 will be proved in Section 4, while Theorem 2 will be proved in Section 5 . We conclude with 5 conjectures. Conjecture 2-5 are based on empirical evidence from section 3. Erickson's last conjecture, E5, are refined into conjecture 3 and 4.

\section{Background}

To be able to understand the present article, the readers need a minimum knowledge of combinatorial game theory, that can be found in [1]. In particular, readers should be familiar with the notions of value of a game and sum games.

We recall the notions of the sum and inequality of two games, the dominated options rule, and the bypass reversible move rule, from [1, pp. 33, 62-64].

\subsection{Sum and Inequality of two games}

Let $G=\left\{G^{L} \mid G^{R}\right\}$, and let $H=\left\{H^{L} \mid H^{R}\right\}$. Then $G+H:=\left\{G^{L}+H, G+H^{L} \mid\right.$ $\left.G^{R}+H, G+H^{R}\right\}$.

$G \geq 0$ if Right goes first and Left wins.

$G \leq 0$ if Left goes first and Right wins.

$G \geq H \Longleftrightarrow G+(-H) \geq 0$.

\subsection{Dominated options rule}

Let $G=\{A, B, C, \cdots \mid D, E, F, \ldots\}$.

If $A \geq B$ and $D \geq E$ then $G=\{A, C, \cdots \mid E, F, \ldots\}$.

\subsection{Bypassing right's reversible move rule}

$G=H$ if $D^{L} \geq G$ (see Figure 1$)$.

In general, rule 2.2 and 2.3 are used to simplify values of a game into the canonical form. All the rules above will be used in the proofs of Theorem 1 and 2 . We use rule 2.2 and 2.3 implicitly to show how one simplifies a recursive calculation. 

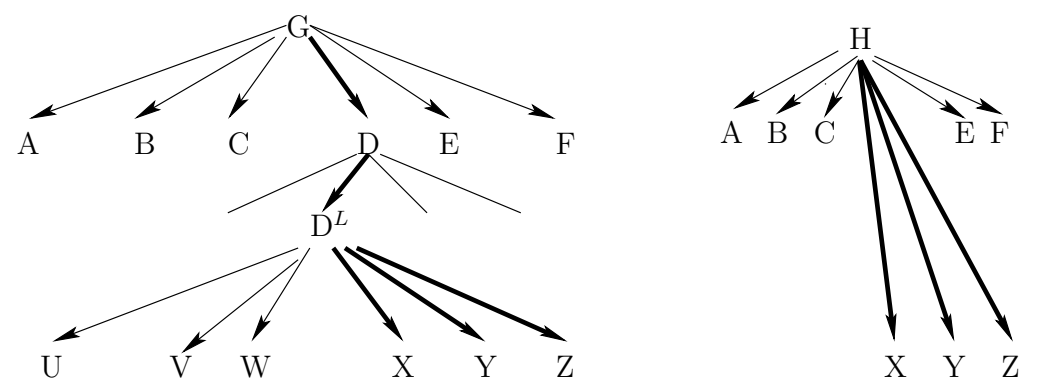

Figure 1: Bypass reversible move rule.

\subsection{Special notation}

The only special notations we use are $*(=\{0 \mid 0\})$ and $n *(=\{n \mid n\})$. We will not use any shorthand notation like $\uparrow$, , etc.

\section{Empirical Evidence}

We present the values of some starting positions in this section. We have a fast program written in Java to compare the values of two positions $(=,>,<, \|$ (not comparable) ) by using the definition of the sum of two games in 2.1. This program does not calculate the value of the sum of two games. The author's brother and the author wrote this program originally to check the value of the game of the form $\mathrm{T}^{a} \square^{b} \mathrm{~F}^{a}$. It works well with the positions that have a simple value. We compare $\mathrm{T}^{a} \square^{b} \mathrm{~F}^{a}$ with 0 or $*$ and hope that the two values are equal. All values of $\mathrm{T}^{a} \square^{b} \mathrm{~F}^{a}$ that we were able to calculate are 0 or $*$ except the column $b=2$ which will be proved to be infinitesimal when $a \geq 4$. We present the tables here.

\subsection{Values for $\mathrm{T}^{a} \square^{b} \mathrm{~F}^{a}$}

\begin{tabular}{|c|c|c|c|c|c|c|c|c|c|c|c|c|c|c|c|c|c|c|c|c|}
\hline$a \backslash b$ & 1 & 2 & 3 & 4 & 5 & 6 & 7 & 8 & 9 & 10 & 11 & 12 & 13 & 14 & 15 & 16 & 17 & 18 & 19 & 20 \\
\hline 1 & $*$ & 0 & $*$ & 0 & $*$ & 0 & $*$ & 0 & $*$ & 0 & $*$ & 0 & $*$ & 0 & $*$ & 0 & $*$ & 0 & $*$ & 0 \\
2 & $*$ & $*$ & $*$ & $*$ & 0 & 0 & $*$ & 0 & 0 & 0 & 0 & 0 & $*$ & 0 & 0 & 0 & 0 & 0 & $*$ & 0 \\
3 & $*$ & $\pm \frac{1}{8}$ & 0 & $*$ & 0 & $*$ & 0 & 0 & 0 & 0 & 0 & 0 & 0 & $*$ & 0 & 0 & 0 & 0 & 0 & 0 \\
4 & $*$ & $\mathrm{~N}$ & $*$ & 0 & 0 & 0 & 0 & $*$ & $*$ & 0 & 0 & 0 & 0 & 0 & 0 & 0 & 0 & 0 & 0 & 0 \\
5 & $*$ & $\mathrm{~N}$ & $*$ & $*$ & $*$ & 0 & $*$ & 0 & 0 & 0 & & 0 & & & & & & & & \\
6 & $*$ & $\mathrm{~N}$ & $*$ & $*$ & $*$ & $*$ & $*$ & & & & & & & & & & & & & \\
7 & $*$ & $\mathrm{~N}$ & $*$ & $*$ & $*$ & & & & & & & & & & & & & & & \\
8 & $*$ & $\mathrm{~N}$ & $*$ & $*$ & $*$ & & & & & & & & & & & & & & & \\
9 & $*$ & $\mathrm{~N}$ & $*$ & $*$ & & & & & & & & & & & & & & & & \\
10 & $*$ & $\mathrm{~N}$ & $*$ & $*$ & & & & & & & & & & & & & & & & \\
\hline
\end{tabular}

\section{Remarks:}


1. For $b$ where $21 \leq b \leq 103, \mathrm{~T}^{2} \square^{b} \mathrm{~F}^{2}=0$ except $b=25,31,37,43,49,55,61,67,73,79$, $85,91,97,103$ which are $*$.

2. For $b$ where $21 \leq b \leq 53, \mathrm{~T}^{3} \square^{b} \mathrm{~F}^{3}=0$ except $b=29$ which is $*$.

3. The notation $\mathrm{N}$ stands for an infinitesimal. See [2, p.307] for some of these values. These values will be proved to be infinitesimal in Section 5 .

\subsection{Values for $\mathrm{T}^{a+1} \square^{b} \mathrm{~F}^{a}$}

\begin{tabular}{|c|c|c|c|c|c|}
\hline$a \backslash b$ & 1 & 2 & 3 & 4 & 5 \\
\hline 1 & $\left\{\frac{1}{2} \mid 0\right\}$ & 1 & $2 *$ & 3 & $4 *$ \\
2 & $*$ & $\left\{\frac{1}{2} \mid 0\right\}$ & $1 *$ & $2 *$ & 3 \\
3 & $*$ & $\{1 * \mid\{* \mid 0\}\}$ & $\left\{1 \mid\left\{\frac{1}{2} \mid 0\right\}\right\}$ & $1 *$ & 2 \\
4 & $*$ & $\{\{2 \mid 1\} \mid\{* \mid-1\}\}$ & $1 *$ & $\left\{\{2 * \mid 1 *\} \mid\left\{\frac{1}{2} \mid 0\right\}\right\}$ & 1 \\
5 & $*$ & $\{\{3 \mid 1\} \mid\{* \mid-2\}\}$ & $1 *$ & $1 *$ & $\frac{5}{4}<V<2$ \\
6 & $*$ & $\{\{4 \mid 1\} \mid\{* \mid-3\}\}$ & $1 *$ & $1 *$ & $2 *$ \\
7 & $*$ & $\{\{5 \mid 1\} \mid\{* \mid-4\}\}$ & $1 *$ & $1 *$ & $\| 2$ \\
8 & $*$ & $\{\{6 \mid 1\} \mid\{* \mid-5\}\}$ & $1 *$ & $1 *$ & $\| 2$ \\
\hline
\end{tabular}

\begin{tabular}{|c|c|c|c|c|c|c|c|c|c|c|c|}
\hline$a \backslash b$ & 6 & 7 & 8 & 9 & 10 & 11 & 12 & 13 & 14 & 15 & 16 \\
\hline 1 & 5 & $6 *$ & 7 & $8 *$ & 9 & $10 *$ & 11 & $12 *$ & 13 & $14 *$ & 15 \\
2 & $\frac{7}{2}$ & $5 *$ & $\frac{11}{2}$ & $\frac{13}{2}$ & $\frac{15}{2}$ & $\frac{17}{2}$ & $\frac{19}{2}$ & $\frac{41}{4}$ & $\frac{23}{2}$ & $12 *$ & 13 \\
3 & $\frac{11}{4}$ & $\frac{15}{4}$ & $\frac{9}{2}$ & & & & & & & & \\
4 & 2 & $\frac{5}{2}<V<3$ & & & & & & & & & \\
5 & 1 & $\frac{5}{2}<V<3$ & & & & & & & & & \\
6 & $1<V<2$ & $2<$ and $\| 3$ & & & & & & & & & \\
\hline
\end{tabular}

\subsection{Values for $\mathrm{T}^{a+2} \square^{b} \mathrm{~F}^{a}$}

\begin{tabular}{|c|c|c|c|c|c|}
\hline$a \backslash b$ & 1 & 2 & 3 & 4 & 5 \\
\hline 1 & $\{\{1 \mid 1\} \mid 0\}$ & 2 & $4 *$ & 6 & $8 *$ \\
2 & $*$ & $\left\{\frac{3}{2} \mid 0\right\}$ & $2 *$ & $4 *$ & $\frac{95}{16}$ \\
3 & $*$ & $\{2 * \mid\{* \mid 0\}\}$ & $\frac{3}{2}$ & $\left\{\left\{\frac{5}{2} \mid 2\right\} \mid 2\right\}$ & 4 \\
4 & $*$ & $\{\{3 \mid 2\} \mid\{* \mid-1\}\}$ & $2 *$ & $\left\{\{4 * \mid 2 *\} \mid\left\{\frac{3}{2} \mid 1\right\}\right\}$ & 3 \\
5 & $*$ & $\{\{4 \mid 2\} \mid\{* \mid-2\}\}$ & $2 *$ & $2 *$ & $3<V<4$ \\
6 & $*$ & $\{\{5 \mid 2\} \mid\{* \mid-3\}\}$ & $2 *$ & $2 *$ & $\| 4$ \\
7 & $*$ & $\{\{6 \mid 2\} \mid\{* \mid-4\}\}$ & $2 *$ & $2 *$ & \\
\hline
\end{tabular}

\begin{tabular}{|c|c|c|c|c|c|c|c|}
\hline$a \backslash b$ & 6 & 7 & 8 & 9 & 10 & 11 & 12 \\
\hline 1 & 10 & $12 *$ & 14 & $16 *$ & 18 & $20 *$ & 22 \\
2 & $\frac{15}{2}$ & $9 *$ & $\{11 \mid 11 *\}$ & 12 & $\frac{29}{2}$ & $15 *$ & $17 *$ \\
3 & $\left\{\frac{11}{2} \mid \frac{41}{8}\right\}$ & 7 & & & & & \\
4 & 4 & $5 *$ & & & & & \\
5 & 3 & 5 & & & & & \\
\hline
\end{tabular}




\subsection{Values for $\mathrm{T}^{a+3} \square^{b} \mathrm{~F}^{a}$}

\begin{tabular}{|c|c|c|c|c|c|c|c|c|}
\hline$a \backslash b$ & 1 & 2 & 3 & 4 & 5 & 6 & 7 & 8 \\
\hline 1 & $\{\{2 \mid 1\} \mid 0\}$ & 3 & $6 *$ & 9 & $12 *$ & 15 & $18 *$ & 21 \\
2 & $*$ & $\left\{\frac{5}{2} \mid 0\right\}$ & $3 *$ & $\left\{6 \mid \frac{11}{2}\right\}$ & $\left\{\frac{17}{2} \mid 8\right\}$ & $11 *$ & 13 & $\frac{31}{2}$ \\
3 & $*$ & $\{3 * \mid\{* \mid 0\}\}$ & $\frac{5}{2}$ & $\mathrm{~L}$ & $\frac{41}{8}$ & $\| 8$ & & \\
4 & $*$ & $\{\{4 \mid 3\} \mid\{* \mid-1\}\}$ & $3 *$ & $\frac{5}{2}$ & 5 & $\| \mid 5$ & & \\
5 & $*$ & $\{\{5 \mid 3\} \mid\{* \mid-2\}\}$ & $3 *$ & $\| 3$ & $5<V<6$ & & & \\
6 & $*$ & $\{\{6 \mid 3\} \mid\{* \mid-3\}\}$ & $3 *$ & $3 *$ & & & & \\
\hline
\end{tabular}

Remark: The value of $\mathrm{T}^{6} \square^{4} \mathrm{~F}^{3}$ in Table 3.4 is long. We do not write it out.

Corollary 1. Conjecture E4 is false.

Proof: From the table, $T^{7} \square^{7} F^{6}>2$.

From numerical results above, the author believes there are no patterns in $a$ for positions of the form $\mathrm{T}^{a+k} \square^{a+l} \mathrm{~F}^{a}$ for any fixed $k \geq 1$ and $l \geq 0$.

\section{Positions with one}

In this section we classify all the positions that have one $\square$. We can compute the values of all positions in this class by 8 simple lemmas. In Section 4.1, we explain some notations we use in Theorem 1 and 2. In Section 4.2, we prove one lemma which is also used in Theorem 1 and 2. Finally we prove Theorem 1 in Section 4.3.

\subsection{Convention.}

For $G \leq 0$, we want to show that Right can win when Left moves first. We show that for each of the possible Left choices, Right has a response that wins the game.

Similarly, we show $G \leq H$ by considering $G-H \leq 0$. Therefore we want to show that for all the possible Left moves in the game $G$ or Right moves in the game $H$, there is a winning reply by Right in the game $G$ or Left in the game $H$.

Below is an example of the notation we use in this paper.

Example: To show: $\quad \mathrm{T}^{a} \mathrm{~F} \square \mathrm{T}^{k} \mathrm{FT} \mathrm{F}^{b} \leq \frac{1}{2}, \quad k \geq 0, a \geq 0, b \geq 1$.

$$
\stackrel{\stackrel{2}{\vec{T}}}{\mathrm{~T}^{a}} \square \mathrm{T}^{k} \mathrm{~F} \stackrel{\frac{1}{\vec{T}}}{\overrightarrow{\mathrm{T}}} \square \mathrm{F}^{b} \leq \stackrel{\frac{3}{1}}{\frac{1}{2}}
$$

Left has three choices to play, moving $\mathrm{T}$ on the left hand side or making a move on the right hand side. The arrows above show the possible moves of Left on the left hand side and Right on the right hand side in an order from 1 to 3. Right could respond to each one of Left's moves as follows:

First choice: Right responds by moving in the left option of $\{0 \mid 1\}$ on the right hand side. This leads to the position:

Case 1: $\mathrm{T}^{a} \mathrm{~F} \square \mathrm{T}^{k} \mathrm{~F} \square \mathrm{TF}^{b} \leq 0$.

Second choice: Right responds by moving the left most F. This leads to the position: 
Case 2: $\mathrm{T}^{a-1} \mathrm{~F} \square \mathrm{T}^{k+1} \mathrm{FT} \square \mathrm{F}^{b} \leq \frac{1}{2}$.

Third choice: Left picks Right option of $\{0 \mid 1\}$ on the right hand side. Right responds by moving the right most $\mathrm{F}$. This leads to the position:

Case 3: $\mathrm{T}^{a} \mathrm{~F} \square \mathrm{T}^{k} \mathrm{FTF} \square \mathrm{F}^{b-1} \leq 1$.

In conclusion, if Left moves as in $\stackrel{i}{\rightarrow}$ it suffices to show Case $i$.

Note: There is no claim of proof in this example.

\subsection{One side Death Leap Principle.}

The proof of this lemma is similar to the proof of DLP [1, p.127].

Lemma 4.1. One side Death Leap Principle (One side DLP): If $X$ is a position with no two consecutive empty squares and the only possible move for Left is a jump, then $X \leq 0$.

Proof: In such positions, Left's moves are necessarily jumps and always clear a space for Right to reply. As a result, if Left moves first, Right can always reply.

Examples: TTF $\square \mathrm{TTF} \square \mathrm{F} \leq 0$ and $\mathrm{TTTF} \square \mathrm{F} \square \mathrm{TF} \leq 0$.

\subsection{Proof of Theorem 1}

Lemma 4.2 is trivial. Lemma 4.3 was first introduced in [1, p.125] and then a generalization was proved in [2]. The rest of the lemmas are new. The proofs are not difficult since both sides have limited choices to make. We will prove them here for completeness. The hardest part of this theorem is the categorization of these positions to the lemmas themselves.

Notations

$O(x)=\{0 \mid x\}$. $O^{a}(x)=O(\ldots(O(O(x)))) a$ times.

$\widetilde{\mathrm{L}}=$ empty or any combination of $\mathrm{T}$ and $\mathrm{F}$ that has $\mathrm{F}$ as its rightmost entry. For example TTFTF.

$\widetilde{\mathrm{R}}=$ empty or any combination of $\mathrm{T}$ and $\mathrm{F}$ that has $\mathrm{T}$ as its leftmost entry. For example TFTTF.

Lemma 4.2. $\widetilde{\mathrm{LT}}{ }^{a} \square=a, a \geq 0$ and $P_{1} \mathrm{FF} \square P_{2}=\square P_{2}$ for any position $P_{1}$ and $P_{2}$.

Lemma 4.3. Death Leap Principle(DLP): Any position with one empty square for which the only possible move for both sides is a jump has value 0.

Example: TFTTF $\square$ TFTFF $=0$.

Lemma 4.4. $\widetilde{\mathrm{LT}} \square \mathrm{F} \widetilde{\mathrm{R}}=*$. 
Proof: $\widetilde{\mathrm{L} T} \square \mathrm{F} \widetilde{\mathrm{R}}=\{\widetilde{\mathrm{L}} \square \mathrm{TFR} \widetilde{\mathrm{R}} \mid \widetilde{\mathrm{LTF}} \square \widetilde{\mathrm{R}}\}$

$=\{0 \mid 0\}$, both Left option and Right option are 0 from DLP.

Lemma 4.5. $\widetilde{\mathrm{LT}^{a}} \square \mathrm{F}^{b} \widetilde{\mathrm{R}}=*, \quad a \geq 2, b \geq 2$.

Proof: We show $\widetilde{\mathrm{L}} \mathrm{T}^{a} \square \mathrm{F}^{b} \widetilde{\mathrm{R}}+\{0 \mid 0\} \leq 0 \ldots$ (1)

$$
\text { and } \widetilde{\mathrm{L}} \mathrm{T}^{a} \square \mathrm{F}^{b} \widetilde{\mathrm{R}}+\{0 \mid 0\} \geq 0 \ldots(2)
$$

Proof of (1): $\quad \widetilde{\mathrm{L}} \stackrel{\overrightarrow{1}}{\overrightarrow{\mathrm{T}}} \square^{b} \widetilde{\mathrm{R}}+\left\{\stackrel{\frac{2}{\overrightarrow{0}}}{0} 0\right\} \leq 0$.

Case 1: $\widetilde{\mathrm{LT}^{a-1}} \mathrm{~F}^{\frac{1}{\overrightarrow{\mathrm{T}}}} \square \mathrm{F}^{b-1} \widetilde{\mathrm{R}}+\{\stackrel{\stackrel{2}{\overrightarrow{0}} \mid}{0} 0\} \leq 0$.

Case 1.1: $\widetilde{\mathrm{LT}^{a-1}} \mathrm{~F} \mathrm{TF}^{b-1} \widetilde{\mathrm{R}} \leq 0$. The left hand side is 0 by DLP.

Case 1.2: $\widetilde{\mathrm{L} T}{ }^{a-1} \mathrm{FTF} \square \mathrm{F}^{b-2} \widetilde{\mathrm{R}} \leq 0$, true by one side DLP.

Case 2: $\widetilde{\mathrm{L} T}{ }^{a} \mathrm{~F} \square \mathrm{F}^{b-1} \widetilde{\mathrm{R}} \leq 0$, true by one side DLP.

Proof of (2): $\quad \widetilde{\mathrm{L}^{a}} \square \mathrm{F}^{b} \widetilde{\mathrm{R}}+\{0 \mid 0\} \geq 0$,

We multiply -1 both sides and apply (1).

Lemma 4.6. $\widetilde{\mathrm{L} T}{ }^{a} \square(\mathrm{TF})^{b}=\left\{a-1 \mid\left(\frac{1}{2}\right)^{b-1}\right\}, \quad a \geq 1, b \geq 1$.

Proof: We use induction on $b$.

Base Case: $b=1$.

$$
\begin{aligned}
\widetilde{\mathrm{LT}^{a}} \square \mathrm{TF} & =\left\{\widetilde{\mathrm{L} T} T^{a-1} \square \mathrm{TTF} \mid \widetilde{\mathrm{L} T}{ }^{a} \mathrm{FT} \square\right. \\
& =\{a-1 \mid 1\}, \text { since } \widetilde{\mathrm{L}} \mathrm{T}^{a} \mathrm{FT} \square=\widetilde{\mathrm{L}} \mathrm{T}^{a} \mathrm{~F} \square+\mathrm{T} \square=0+1 .
\end{aligned}
$$

\section{Induction Step:}

$$
\begin{aligned}
\widetilde{\mathrm{LT}}^{a} \square(\mathrm{TF})^{b} & =\left\{\widetilde{\mathrm{LT}} \mathrm{T}^{a-1} \square \mathrm{T}(\mathrm{TF})^{b} \mid \widetilde{\mathrm{L}} \mathrm{T}^{a} \mathrm{FT} \square(\mathrm{TF})^{b-1}\right\} \\
& =\left\{a-1 \mid\left\{1-1 \mid\left(\frac{1}{2}\right)^{b-2}\right\}\right\}, \text { Right option is from induction step. } \\
& =\left\{a-1 \mid\left(\frac{1}{2}\right)^{b-1}\right\} .
\end{aligned}
$$

Lemma 4.7. $\widetilde{\mathrm{LT}}{ }^{a} \square \mathrm{F}(\mathrm{TF})^{b}=\left\{\left\{a-2 \mid\left(\frac{1}{2}\right)^{b}\right\} \mid 0\right\}, \quad a \geq 2, b \geq 0$.

Proof: $\widetilde{\mathrm{L}} \mathrm{T}^{a} \square \mathrm{F}(\mathrm{TF})^{b}=\left\{\widetilde{\mathrm{L}} \mathrm{T}^{a-1} \square(\mathrm{TF})^{b+1} \mid \widetilde{\mathrm{L}} \mathrm{T}^{a} \mathrm{~F} \square(\mathrm{TF})^{b}\right\}$

$$
=\left\{\left\{a-2 \mid\left(\frac{1}{2}\right)^{b}\right\} \mid 0\right\} \text {. }
$$

Left option is true by Lemma 4.6. Right option is true by DLP.

Lemma 4.8. $\widetilde{\mathrm{LT}}{ }^{a} \square(\mathrm{TF})^{b} \mathrm{TF}^{c} \widetilde{\mathrm{R}}=\left\{a-1 \mid\left(O^{b}\left(\widetilde{\mathrm{LT}}{ }^{a} \mathrm{~F}(\mathrm{TF})^{b} \mathrm{~T} \mathrm{~F}^{c-1} \widetilde{\mathrm{R}}\right)\right\}, \quad a \geq 1, b \geq 0, c \geq\right.$ 2 .

Proof: We use induction on $b$. We show Left option is $a-1$ and Right option is $O^{b}(x)$ where $x:=\widetilde{\mathrm{L} T}{ }^{a} \mathrm{~F}(\mathrm{TF})^{b} \mathrm{~T} \mathrm{~F}^{c-1} \widetilde{\mathrm{R}}$.

In other words, Left option: $\widetilde{\mathrm{L}} \mathrm{T}^{a-1} \square \mathrm{T}(\mathrm{TF})^{b} \mathrm{TF}^{c} \widetilde{\mathrm{R}}=a-1$.

Right option: $\widetilde{\mathrm{L} T}{ }^{a} \mathrm{FT} \square \mathrm{F}^{c-1} \widetilde{\mathrm{R}}=x$, for $b=0$ and $\widetilde{\mathrm{LT}}{ }^{a} \mathrm{FT} \square(\mathrm{TF})^{b-1} \mathrm{TF}^{c} \widetilde{\mathrm{R}}=O^{b}(x)$, for $b \geq 1$.

Left option is trivially true. We prove only Right option.

Base Case: $b=0$ and $b=1$

$b=0$, the statement is trivial.

$b=1, \widetilde{\mathrm{LT}^{a}} \mathrm{FT} \mathrm{TF}^{c} \widetilde{\mathrm{R}}=\left\{\widetilde{\mathrm{LT}^{a}} \mathrm{~F}^{\mathrm{T}} \mathrm{TTF}^{c} \widetilde{\mathrm{R}} \mid \widetilde{\mathrm{LT}^{a}} \mathrm{FTFT} \mathrm{F}^{c-1} \widetilde{\mathrm{R}}\right\}$ 
Induction Step: $b \geq 2$

$$
\begin{aligned}
& =\{0 \mid x\}, \text { Left option is true by DLP. } \\
& =O(x) .
\end{aligned}
$$

$$
\begin{aligned}
& \widetilde{\mathrm{L}}{ }^{a} \mathrm{FT} \square(\mathrm{TF})^{b-1} \mathrm{TF}^{c} \widetilde{\mathrm{R}}=\left\{\widetilde{\mathrm{LT}}{ }^{a} \mathrm{~F} \square \mathrm{T}(\mathrm{TF})^{b-1} \mathrm{TF}^{c} \widetilde{\mathrm{R}} \mid \widetilde{\mathrm{L}} \mathrm{T}^{a} \mathrm{FTFT} \square(\mathrm{TF})^{b-2} \mathrm{TF}^{c} \widetilde{\mathrm{R}}\right\} \\
& =\left\{0 \mid \widetilde{\mathrm{L}}^{\prime} \mathrm{T} \square(\mathrm{TF})^{b-2} \mathrm{TF}^{c} \widetilde{R}\right\} \text { where } \widetilde{\mathrm{L}}^{\prime}:=\widetilde{\mathrm{L} T}{ }^{a} \mathrm{FTF}, \\
& \text { Left option is true by DLP. } \\
& =\left\{0 \mid\left\{0 \mid O^{b-2}\left(\widetilde{\mathrm{L}}{ }^{\prime} \mathrm{TF}(\mathrm{TF})^{b-2} \mathrm{~T} \mathrm{~F}^{c-1} \widetilde{\mathrm{R}}\right)\right\}\right\} \text {, by induction on } b \text {. } \\
& =\left\{0 \mid\left\{0 \mid O^{b-2}\left(\widetilde{\mathrm{LT}}{ }^{a} \mathrm{~F}(\mathrm{TF})^{b} \mathrm{~T} \mathrm{~F}^{c-1} \widetilde{\mathrm{R}}\right)\right\}\right\} \\
& =\left\{0 \mid\left\{0 \mid O^{b-2}(x)\right\}\right\} \\
& =O^{b}(x) \text {. }
\end{aligned}
$$

Lemma 4.9. $\widetilde{\mathrm{LT}}{ }^{a} \square \mathrm{F}(\mathrm{TF})^{b} \mathrm{TF}^{c} \widetilde{\mathrm{R}}=\left\{\left\{a-2 \mid O^{b+1}\left(\widetilde{\mathrm{LT}} \mathrm{T}^{a-1} \mathrm{~F}(\mathrm{TF})^{b+1} \mathrm{~T} \mathrm{~F}^{c-1} \widetilde{\mathrm{R}}\right)\right\} \mid 0\right\}, \quad a \geq$ $2, b \geq 0, c \geq 2$.

Proof: $\widetilde{\mathrm{L}} \mathrm{T}^{a} \square \mathrm{F}(\mathrm{TF})^{b} \mathrm{TF}^{c} \widetilde{\mathrm{R}}=\left\{\widetilde{\mathrm{LT}}{ }^{a-1} \square(\mathrm{TF})^{b+1} \mathrm{TF}^{c} \widetilde{\mathrm{R}} \mid \widetilde{\mathrm{L}} \mathrm{T}^{a} \mathrm{~F} \square(\mathrm{TF})^{b} \mathrm{TF}^{c} \widetilde{\mathrm{R}}\right\}$

$$
\begin{aligned}
& =\left\{\left\{a-2 \mid O^{b+1}\left(\widetilde{\mathrm{LT}}{ }^{a-1} \mathrm{~F}(\mathrm{TF})^{b+1} \mathrm{~T}^{2} \mathrm{~F}^{c-1} \widetilde{\mathrm{R}}\right)\right\} \mid 0\right\} . \\
& \text { Left option is by Lemma 4.8. Right option is by DLP. }
\end{aligned}
$$

Example: $\mathrm{T}^{a} \square \mathrm{F}(\mathrm{TF})^{b} \mathrm{TF}^{2}=\left\{\left\{a-2 \mid O^{b+1}(*)\right\} \mid 0\right\}, \quad a \geq 2, b \geq 0$.

Remark: The position $x$ in Lemma 4.8 and 4.9 can be recursively computed using Lemma $4.4,4.7$ and 4.9 .

\section{Positions $\mathrm{T}^{a} \square \square \mathrm{F}^{a}$}

In this section, we show $\mathrm{T}^{a} \square \square \mathrm{F}^{a}$ is an infinitesimal for $a \geq 4$. The observation comes from Table 3.1 when $b=2$. We first state the following lemma.

Lemma 5.1. For any fixed integer $n \geq 3, \widetilde{\mathrm{LT}^{a}} \mathrm{~F} \square \mathrm{T}^{k} \mathrm{FT} \square \mathrm{F}^{b} \leq \frac{1}{2^{n}}, k \geq 0, a \geq 0, b \geq 1$.

Proof: We use induction on $a$.

Base Case: $a=0, \square \mathrm{T}^{k} \mathrm{~F} \underset{\mathrm{T}}{\overrightarrow{\mathrm{T}}} \square \mathrm{F}^{b} \leq \frac{2}{2^{n}}$.

Case 1: $\square \mathrm{T}^{k} \mathrm{~F} \square \mathrm{TF}^{b} \leq 0$, true by one side DLP.

Case 2: $\square \mathrm{T}^{k} \mathrm{FTF} \square \mathrm{F}^{b-1} \leq \frac{2}{2^{n}}$. The left hand side is $\leq 0$ by one side DLP.

Induction Step: $\widetilde{\mathrm{L}} \stackrel{\stackrel{2}{\vec{T}}}{a} \mathrm{~F} \square \mathrm{T}^{k} \mathrm{~F} \stackrel{\frac{1}{\vec{T}}}{\overrightarrow{\mathrm{T}}} \square \mathrm{F}^{b} \leq \frac{\frac{3}{1}}{2^{n}}$.

Case 1: $\widetilde{\mathrm{L}} \mathrm{T}^{a} \mathrm{~F} \square \mathrm{T}^{k} \mathrm{~F} \square \mathrm{TF}^{b} \leq 0$, true by one side DLP.

Case 2: $\widetilde{\mathrm{LT}^{a-1}} \mathrm{~F} \square \mathrm{T}^{k+1} \mathrm{FT} \square \mathrm{F}^{b} \leq \frac{1}{2^{n}}$, true by induction.

Case 3: $\widetilde{\mathrm{LT}^{a}} \mathrm{~F} \square \mathrm{T}^{k} \mathrm{FTF} \square \mathrm{F}^{b-1} \leq \frac{2}{2^{n}}$. The left hand side is $\leq 0$ by one side DLP.

We are now ready to prove the main theorem.

\section{Proof of Theorem 2.}

By symmetry we only need to show that, for any fixed integer $n \geq 3, \mathrm{~T}^{a} \square \square \mathrm{F}^{a} \leq \frac{1}{2^{n}}, a \geq$ 4 . 


$$
\begin{aligned}
& \stackrel{\stackrel{I}{\rightarrow}}{\mathrm{T}^{a}} \square \square \mathrm{F}^{a} \leq \stackrel{\stackrel{I I}{\leftarrow}}{2^{n}} . \\
& \text { I) } \stackrel{\stackrel{2}{\vec{a}}}{\mathrm{~T}^{-1}} \square \stackrel{\frac{1}{\mathrm{~T}}}{\mathrm{~T}} \square \mathrm{F}^{a-1} \leq \frac{\stackrel{3}{\frac{1}{2}}}{2^{n}} \\
& \text { II) } \stackrel{\stackrel{1}{\rightarrow}}{\mathrm{T}} \square \mathrm{F} \square \mathrm{F}^{a-1} \leq \frac{\stackrel{2}{\leftarrow}}{2^{n}}
\end{aligned}
$$

I) Case 1: $\stackrel{\stackrel{1}{a}}{\mathrm{~T}^{-1}} \square \mathrm{F} \square \mathrm{TF}^{a-1} \leq \stackrel{\stackrel{2}{\frac{1}{2}}}{2^{n}}$

Case 1.1: $\mathrm{T}^{a-2} \mathrm{~F} \stackrel{\frac{1}{\mathrm{~T}}}{\square} \square \mathrm{TF}^{a-1} \leq \stackrel{\stackrel{2}{\frac{1}{2}}}{2^{n}}$

Case 1.1.1: $\mathrm{T}^{a-2} \mathrm{~F} \square \mathrm{TFT} \square \mathrm{F}^{a-2} \leq \frac{1}{2^{n}}$, true by Lemma 5.1.

Case 1.1.2: $\mathrm{T}^{a-2} \mathrm{~F} \stackrel{\stackrel{2}{\mathrm{~T}}}{\square} \square \mathrm{F} \stackrel{\stackrel{1}{\mathrm{~T}}}{\square} \square \mathrm{F}^{a-2} \leq \frac{\stackrel{3}{\frac{2}{2}}}{2^{n}}$

Case 1.1.2.1: $\mathrm{T}^{a-2} \mathrm{~F} \stackrel{\stackrel{1}{\mathrm{~T}}}{\mathrm{~F}} \square \square \mathrm{TF}^{a-2} \leq \frac{\stackrel{2}{\frac{2}{2}}}{2^{n}}$

Case 1.1.2.1.1: $\square \mathrm{T} \square \mathrm{TF}^{a-2} \leq \frac{2}{2^{n}}$.

The value of the left hand side is $\{0 \mid\{0 \mid\{-1 \mid 5-a\}\}\}$ (refer to ClassA22 (Class A with two $\square$ and two F) in [6]) which confirms the case above.

Case 1.1.2.1.2: $\mathrm{T}^{a-2} \mathrm{FTF} \square \mathrm{FT} \square \mathrm{F}^{a-3} \leq \frac{4}{2^{n}}$, true by Lemma 5.1 which $\widetilde{\mathrm{L}}=\mathrm{T}^{a-2} \mathrm{~F}, a^{\prime}=1, k^{\prime}=0$ and $b^{\prime}=a-3$

(as notations used in Lemma 5.1).

Case 1.1.2.2: $\mathrm{T}^{a-2} \mathrm{~F} \square \mathrm{TFTF} \square \mathrm{F}^{a-3} \leq \frac{2}{2^{n}}$, true by one side DLP.

Case 1.1.2.3: $\mathrm{T}^{a-2} \mathrm{~F} \stackrel{\stackrel{2}{\mathrm{~T}}}{\square} \square \stackrel{\stackrel{1}{\rightarrow}}{\mathrm{T}} \mathrm{F} \square \mathrm{F}^{a-3} \leq \frac{\stackrel{3}{4}}{2^{n}}$

Case 1.1.2.3.1: $\mathrm{T}^{a-2} \mathrm{FT} \square \mathrm{F} \square \mathrm{FTF}^{a-3} \leq 0$ since $\mathrm{T}^{a-2} \mathrm{~F} \square \mathrm{TFF} \square \mathrm{TF}^{a-3} \leq 0$, true by one side DLP.

Case 1.1.2.3.2: $\mathrm{T}^{a-2} \mathrm{~F} \square \mathrm{TFTFF} \square \mathrm{F}^{a-4} \leq \frac{4}{2^{n}}$, true by one side DLP.

Case 1.1.2.3.3: $\mathrm{T}^{a-2} \mathrm{FTF} \square \mathrm{TF} \square \mathrm{F}^{a-3} \leq \frac{8}{2^{n}}$, true by one side DLP.

Case 1.2: $\stackrel{\stackrel{2}{\rightarrow}}{\mathrm{T}^{a-1}} \square \mathrm{FF} \stackrel{\stackrel{1}{\rightarrow}}{\mathrm{T}} \square \mathrm{F}^{a-2} \leq \frac{\stackrel{3}{\frac{2}{2}}}{2^{n}}$

Case 1.2.1: $\mathrm{T}^{a-1} \mathrm{~F} \square \mathrm{F} \square \mathrm{TF}^{a-2} \leq \frac{2}{2^{n}}$, true by one side DLP.

Case 1.2.2: $\mathrm{T}^{a-2} \mathrm{FT} \square \mathrm{FT} \square \mathrm{F}^{a-2} \leq \frac{2}{2^{n}}$. This is the case 1.1.2.

Case 1.2.3: $\mathrm{T}^{a-1} \mathrm{~F} \square \mathrm{FT} \square \mathrm{F}^{a-2} \leq \frac{4}{2^{n}}$, true by Lemma 5.1 .

Case 2: $\mathrm{T}^{a-2} \square \mathrm{TTFF} \square \mathrm{F}^{a-2} \leq \frac{1}{2^{n}}$. The left hand side is 0 .

Case 3: $\stackrel{\stackrel{1}{\vec{a}}}{\mathrm{~T}^{a-1}} \square \mathrm{TFF} \square \mathrm{F}^{a-2} \leq \stackrel{\stackrel{2}{\frac{2}{2}}}{2^{n}}$

Case 3.1: $\mathrm{T}^{a-2} \square \mathrm{TTFF} \square \mathrm{F}^{a-2} \leq 0$. See Case 2.

Case 3.2: $\mathrm{T}^{a-1} \mathrm{FT} \square \mathrm{F} \square \mathrm{F}^{a-2} \leq \frac{4}{2^{n}}$. The left hand side is $\leq 0$, since the only possible left move leads to $\mathrm{T}^{a-1} \mathrm{FFT} \square \square \mathrm{F}^{a-2}=3-a$ (refer to the value to ClassA21 in [6]).

II) Case 1: $\mathrm{T}^{a-1} \square \mathrm{TFF} \square \mathrm{F}^{a-2} \leq \frac{2}{2^{n}}$. This is I) case 3 .

Case $2: \stackrel{\stackrel{1}{\rightarrow}}{\mathrm{T}^{a}} \square \mathrm{FF} \square \mathrm{F}^{a-2} \leq \frac{2}{\frac{4}{2^{n}}}$ 
Case 2.1: $\mathrm{T}^{a-1} \mathrm{FT} \square \mathrm{F} \square \mathrm{F}^{a-2} \leq \frac{4}{2^{n}}$. This is I) case 3.2.

Case 2.2: $\mathrm{T}^{a} \mathrm{~F} \square \mathrm{F} \square \mathrm{F}^{a-2} \leq \frac{8}{2^{n}}$, true by one side DLP.

\section{New Conjectures and Future Work}

We believe there are still a lot of nice patterns and conjectures in this game that we overlooked. We will have more information when RAM gets cheaper and Maple gets faster. We make the following conjectures.

\section{Conjectures:}

T1: Assume $a \geq 1, b \geq 0, L \geq 0$ and $R \geq 0$

$\mathrm{T} 1.1: \square^{R} \mathrm{~T}^{a} \square^{b} \mathrm{~F} \square^{R}= \begin{cases}\{a-2 \mid 1\} \mid 0\} & \text { if } R=0 \text { and } b=1 \\ (a-1)(b-1+R) & \text { if } b \text { is even and }(R, b) \neq(0,0) \\ (a-1)(b-1+R) * & \text { if } b \text { is odd and }(R, b) \neq(0,1)\end{cases}$

T1.2: For $R \geq 1, \quad \square^{R-1} \mathrm{~T}^{a} \square^{b} \mathrm{~F} \square^{R}= \begin{cases}(a-1)(b-1+R) & \text { if } b \text { is even } \\ \frac{1}{2}+(a-1)(b-1+R) & \text { if } b \text { is odd }\end{cases}$

T1.3: For $R-L \geq 2, \square^{L} \mathrm{~T}^{a} \square^{b} \mathrm{~F} \square^{R}=(R-L-1)+(a-1)(b-1+R)$.

$\mathrm{T} 2$ : For $a \geq 7, \mathrm{TT}^{a} \mathrm{FF}= \begin{cases}* & \text { when } a=7+6 n, \quad n \geq 0 \\ 0 & \text { otherwise. }\end{cases}$

T3: $\mathrm{T}^{a} \square^{b} \mathrm{~F}^{a}=*$ for any $a>b>0$, except for $b=2$.

T4: $\mathrm{T}^{a} \square^{b} \mathrm{~F}^{a}=0$ or $*$ for any $b \geq a>0$.

T5: For any fixed integer $C \geq 3, \exists a_{0}$ such that $\mathrm{T}^{C} \square^{a} \mathrm{~F}^{C}=0$ for all $a \geq a_{0}$.

Future Work:

1) Categorize all the positions that have exactly one F (general class B1) (conjecture T1 might be a good start).

\section{Acknowledgements}

We thank our trainer Doron Zeilberger for always supporting and motivating. We also thank our twin brother Thotsaphon Thanatipanonda for writing the fast program in Java that found the counterexample to Conjecture E4. Last but not least we thank an anonymous referee for her/his comments that greatly improved this paper. 


\section{References}

[1] Elwyn Berlekamp, John Conway, and Richard Guy, Winning Ways for your Mathematical Plays, Academic Press, New York, 1982.

[2] Jeff Erickson, New Toads and Frogs Results, Game of No Chance, 1996.

[3] Jesse Hull, personal website, http://compgeom.cs.uiuc.edu/ jeffe/pubs/toads.html

[4] Thotsaporn "Aek" Thanatipanonda, Doron Zeilberger, A Symbolic Finite-state approach for Automated Proving of Theorems in Combinatorial Game Theory, J. of Difference Equations and Applications 15(2009), 111-118.

[5] Thotsaporn "Aek" Thanatipanonda, Three Results of Combinatorial Game Toads and Frogs, preprint, http://www.risc.jku.at/people/tthanati/.

[6] Thotsaporn "Aek" Thanatipanonda, Library values for Toads and Frogs, http://www.risc.jku.at/people/tthanati/FiniteState.html. 